\title{
Intra-molecular and Intermolecular Proteolysis Activity of p67
}

\author{
Bansidhar Datta \\ Department of Chemistry and Biochemistry, Kent State University, Kent, OH \\ bdatta@kent.edu
}

\begin{abstract}
The cellular glycoprotein, p67 binds directly to eukaryotic initiation factor 2 (eIF2) and extracellular signal-regulated kinases 1 and 2 (ERK1/2) and regulates their levels of phosphorylation. The cDNA sequences for p67 homologs were cloned from various species such as yeast, insects, plants, mouse, rat, and human. The rat cDNA was extensively characterized by detailed mutagenesis accompanied with a series of biochemical analysis in in vitro, ex vivo, and in vivo assays. These assays identified a series of functional domains/motifs that play important roles in p67's multi-faceted activities such as blocking phosphorylation of eIF2, ERK1/2, and possibly other unknown kinases and intra-molecular (auto-proteolysis) and intermolecular proteolysis activity. Auto-proteolysis of rat and human p67 generates several peptide fragments. The most stable fragments are p26 that covers $N$-terminal 1-107 amino acid residues and p52 that covers downstream 108-480 amino acid residues. The p26 segment seems to be the business end of the molecule, whose activity is regulated by the p52 segment. In the p52 segment there are at least five conserved amino acid residues - D251, D262, H331, E364, and E459. These amino acids after folding juxtapose together to create a shallow groove, which serves as the substrate-binding pocket and the nearby $H 231$ residue serves as the catalytic site for p67's intraand/or inter-molecular activities to cleave peptide bonds. The target for intra-molecular proteolysis is p67 itself, whereas the inter-molecular targets are cell cycle regulatory proteins, certain cyclins and Cdks, and p21; cell growth, cytoskeleton dynamics, migration, and motility regulatory proteins, PKC, MARCKS, FAK, Cdc42, PAK1, and Akt1/2 (or PKB); eIF2 $\alpha$-specific kinase, PERK; and possibly several other unidentified kinases and phosphor-proteins that are involved in cell signaling. By proteolytic cleaving various proteins/kinases p67 is involved in the regulation of different cellular processes.
\end{abstract}

Abbreviations Used: eIF2, eukaryotic initiation factor 2; eIF2 $\alpha$, the smallest $\alpha$-subunit of eIF2; p67, an eIF2-associated glycoprotein; ERK1/2, extracellular signal-regulated kinases 1 and 2; GST, glutathione-Stransferase; SDS-PAGE, sodium dodecyl sulfate - polyacrylamide gel electrophoresis; HRI, heme-regulated inhibitor; p26, the N-terminal 1-107 amino acid segment of p67; p52, the downstream 108-480 amino acid segment of p67; MARCKS, myristoyl alanine-rich C kinase substrate; PKC, protein kinase C, FAK, focal adhesion kinase, Pak1, p21-activating kinase, Cdc42, a Rho GTPase; p21, a cell cycle inhibitor; Cdks, cyclindependent kinases; and PERK, an eIF2 $\alpha$-specific kinase activated in response to endoplasmic reticulum stress.

Keywords: Auto-proteolytic activity of p67; Inter-molecular proteolytic activity of p67; p67 block mutants, point mutants, and double mutants; dominant negative and dominant positive mutants of p67

\section{INTRODUCTION}

Detailed descriptions of purification, cloning, biochemical characterization, genetic analyses to identify functional domains/sequence motifs, sequence homologies in open reading frames (ORFs), promoter regions, 5' and 3' un-translated regions (UTRs), and proteins from various organisms, multi-faceted activities in in vitro, ex vivo, and in vivo systems, direct binding to eukaryotic initiation factor 2 (eIF2) and extracellular signal-regulated kinases 1 and 2 (ERK1/2), and existence of multiple p67 isoforms have been published (see reviews 1-3). In this review detailed analysis of the autoproteolysis and intermolecular proteolysis activities of $\mathrm{p} 67$ will be discussed.

\section{P67 haS AUTO-PROTEOLYSIS (INTERAMOLECULAR) ACTIVITY - IN VITRO ANALYSIS}

The auto-proteolysis of p67 from both rat and human has been reported recently (4-5). To conclude that rat p67 has auto-proteolytic activity, a series of glutathione-S-transferase (GST)- and His-tagged p67 mutants such as deletion mutants, block mutants, single point mutants, and double mutants were created, expressed in E. coli or in insect cells, purified through affinity chromatography, and analyzed in SDS-PAGE and column chromatographic procedures (4, 6). For GST-fusion proteins, we detected a very low amount of full length GST-p67 instead we detected two major closely migrating degraded 
fusion proteins running between $36 \mathrm{kDa}$ and $52 \mathrm{kDa}$ markers and several faster migrating degraded proteins (6). Deletion of the N-terminal lysine residue-rich domain II along with the acidic residuerich domain did produce some detectable amount of full-length GST-p67 that is a little better amount than the wild type GST-p67. In contrast, deletion of lysine residue-rich domain I along with the acidic residue-rich domain resulted into much better yield of full-length GST-p67. Deletion of the acidic residue-rich domain however gave slightly lesser amount of full-length GST-p67 as compared to lysine residue-rich domain I deletion mutant. When both lysine residue-rich domains I and II are deleted, we detected much better yield of full-length GST-p67 (Fig. 1, Ref. 6). These results suggest that rat p67 has auto-proteolysis activity and its lysine residue-rich domains I \& II are essential for this activity (6). Since GST-p67 we purified from E. coli possibly did not have any post-translational modifications, its degradation may be triggered by the lack of proper folding.
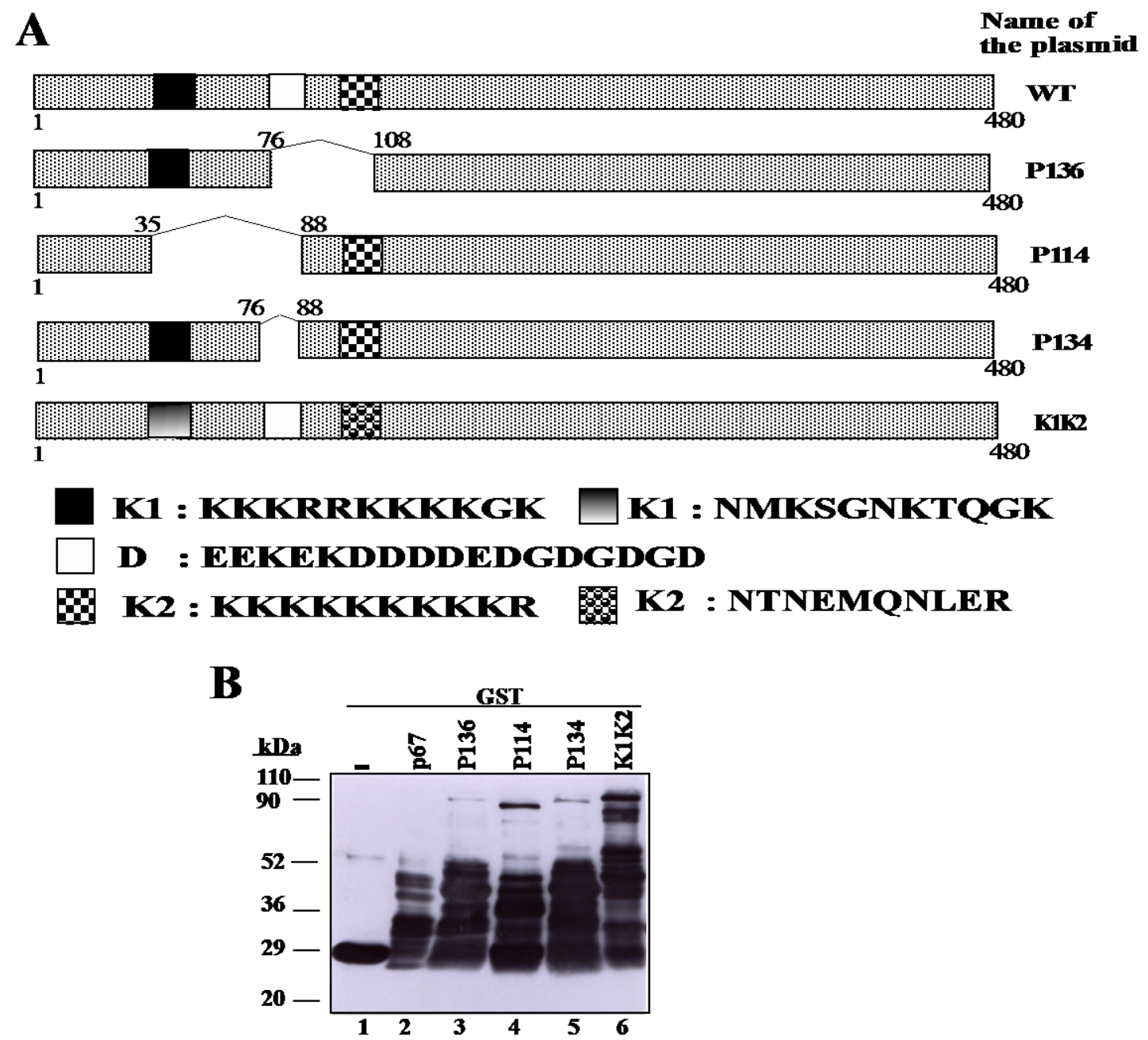

Fig1. Stability of GST-fusions of rat p67 deletion mutants and K1K2 block mutant.

(A) Schematic representations of various p67 deletion mutants and the K1K2 block mutant and sequences of the lysine residue-rich domains I \& II and the acidic residue-rich domain. The solid bar represents the full-length rat p67, filled box represents lysine residue-rich domain I, open box represents the acidic residue-rich domain, and partially filled box represents the lysine residue-rich domain II. (B) The GST protein or its fusions of fulllength rat p67, p67 deletion mutants, P136, P114, and P134, and K1K2 mutant were expressed in E. coli, affinity purified, analyzed on SDS-PAGE followed by Western blotting with a monoclonal antibody specific to GST. Molecular weight markers are shown on the left. This figure is reproduced from Ghosh et. al. (6) with editor's permission.

To eliminate the above possibility we generated a series of His-tagged p67 fusion genes (wild type and mutants) in bacculovirus expression vector, expressed the His-tagged full-length p67 and it's various mutants in Sf21 insect cells (4). These His-tagged proteins were then affinity purified via nickel $\left(\mathrm{Ni}^{2+}\right)$-agarose beads. The activity of the His-tagged full-length rat p67 was examined in phosphorylation assay using three-subunit eIF2 as the substrate for HRI kinase (Fig. 2, Ref. 4). The full-length rat p67 was quite stable and active in protecting the phosphorylation of eIF2 $\alpha$. However, it 
still produced several faster migrating degraded proteins even when stored at $-80^{\circ} \mathrm{C}$ and this degradation was partially inhibited when a cocktail of protease inhibitors was used. Among these degraded proteins, two major polypeptides were identified - one is corresponding to the N-terminal 1107 amino acid segment (also known as p26 based on its migration in SDS-PAGE) and the other is covering the downstream 108-480 amino acid segment (also called p52 based on its migration in SDS-PAGE) of p67 (4). We then generated several Myc-tagged fusion genes corresponding to the full-length rat p67, p67's ${ }_{60} \mathrm{SGTS}_{63}$ mutant $\left({ }_{60} \mathrm{AGAA}_{63}\right), \mathrm{K} 2 / 9 / 1$ mutant ${ }_{98} \mathrm{KKKKKKKKKR}_{107}$ changed to ${ }_{98}$ NTNEMQNLER $\left._{107}\right)$, and K1K2 mutant ${ }_{36}$ KKKRRKKKKGK $_{44}$ and ${ }_{98} \mathrm{KKKKKKKKKR}_{107}$ changed to ${ }_{36} \mathrm{NMKSGNKTQGK}_{44}$ and ${ }_{98} \mathrm{NTNEMQNLER}_{107}$ respectively) in bacculovirus expression vector. These fusion proteins were then purified and analyzed on SDS-PAGE followed by Western blotting using a monoclonal antibody specific to Myc tag (Fig. 3, Ref. 4). Our results show low level of degradation of full-length Myc-p67 ( 2-5\% of full-length p67) and this degradation was very significant ( $\sim 80 \%$ of the full-length mutant) in ${ }_{60} \mathrm{AGAA}_{63}$ mutant. The $\mathrm{K} 2 / 9 / 1$ mutant showed similar level of degradation with full-length $\mathrm{p} 67$ while this degradation was near completely stopped in K1K2 mutant (4). These results suggest that rat p67 indeed has auto-proteolysis activity, which is substantially inhibited when ${ }_{60} \mathrm{SGTS}_{63}$ motif of p67 is O-glycosylated (7-8). The Oglycosylation at this site is required for binding to eIF2 $\alpha$ and protection from phosphorylation by eIF2 $\alpha$-specific kinase, HRI (7-8). In addition, these results also confirm our previous finding that the lysine residue-rich domains are essential for p67's auto-proteolysis.

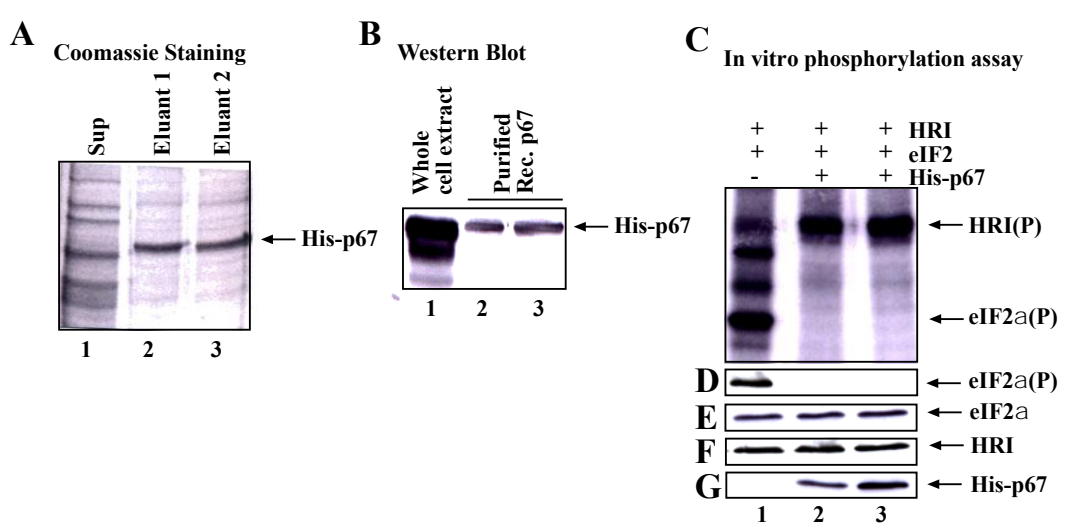

Fig2. Expression and activity of rat p67 purified from bacculovirus-infected insect cells

The full-length rat p67 was sub-cloned into bacculovirus expression vector to generate in-frame-fusion with His-tag. His-p67 was then expressed in Sf21 insect cells, purified through $\mathrm{Ni}^{2+}{ }^{2+}$ agarose beads, and analyzed via SDS-PAGE followed by commassie staining (A). A sample of purified His-p67 was analyzed on Western blot using a monoclonal antibody specific to His-tag (B). The purified His-tag rat p67 was then used in eIF2 $\alpha$ phosphorylation assay following the procedures as described (4). The phosphor-proteins were analyzed by SDS$P A G E$ followed by autoradiography $(C)$, transferred to nitrocellulose membranes followed by Western blot analyses using antibodies specific to the phosphorylated form of eIF $2 \alpha(D)$, total eIF2 $\alpha$ added in the phosphorylation assays (E), the HRI kinase (F), and His-tag to detect His-tagged p67 (G). Lane 1, no p67; lane 2, $0.5 \mu \mathrm{g}$ p67, and lane 3, $1.0 \mu \mathrm{g}$ p67. Sup supernatant.

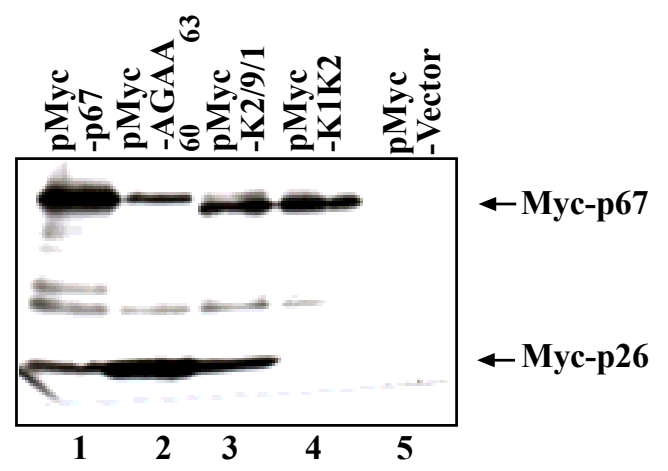

Fig3. Auto-proteolysis of Myc-tagged rat p67

Myc-tagged empty expression vector or its fusions with full-length wild type rat $p 67$ and its mutants, ${ }_{60} A G A A_{63}$, K2/9/1, and K1K2 were transiently expressed in C2C12 myoblasts followed by Western blot analysis using a monoclonal antibody specific to the Myc tag. This figure is reproduced from Datta et. al., (4) with editor's permission. 


\section{In Vivo EVIDENCE FOR InTRAMOlecular ProteOlysis OF P67}

We then analyzed total protein samples from male and female mouse tissues on Western blots. Our results show that p67 is ubiquitously present in all tissues from both male and female mice tested in these experiments, although, its relative amounts in these tissues may vary slightly (9). Another protein $\sim 60 \mathrm{kDa}$ size is present in all tissues used from both male and female mice for these experiments except brain and liver of both male and female mice possibly express this protein in extremely low level. The relative ratios of the p67 and $\sim 60 \mathrm{kDa}$ proteins in tissues they are expressed also vary widely. In our Western blotting experiments we have also detected some faster migrating proteins running around $52 \mathrm{kDa}$ (or p52), $26 \mathrm{kDa}$ (or p26), and others with lower molecular weights in some tissues (9). For example in male mice, p52 is detected in tissues like brain, liver, skeletal muscle, kidney, and testis in varying amounts. Similarly, p26 is detected almost in all tissues tested except spleen and testis where its abundance may be very low (Fig. 4, Ref. 9). In female mice tissues, the relative presence of the faster migrating proteins like p52, p26, and others varies quite a bit (9). For example, both p52 and p26 proteins are present in all female mouse tissues tested in the experiments but their levels are very low as compared to similar male mice tissues. Together, these findings confirm the presence of both p26 and p52 segments of p67 in live mice.

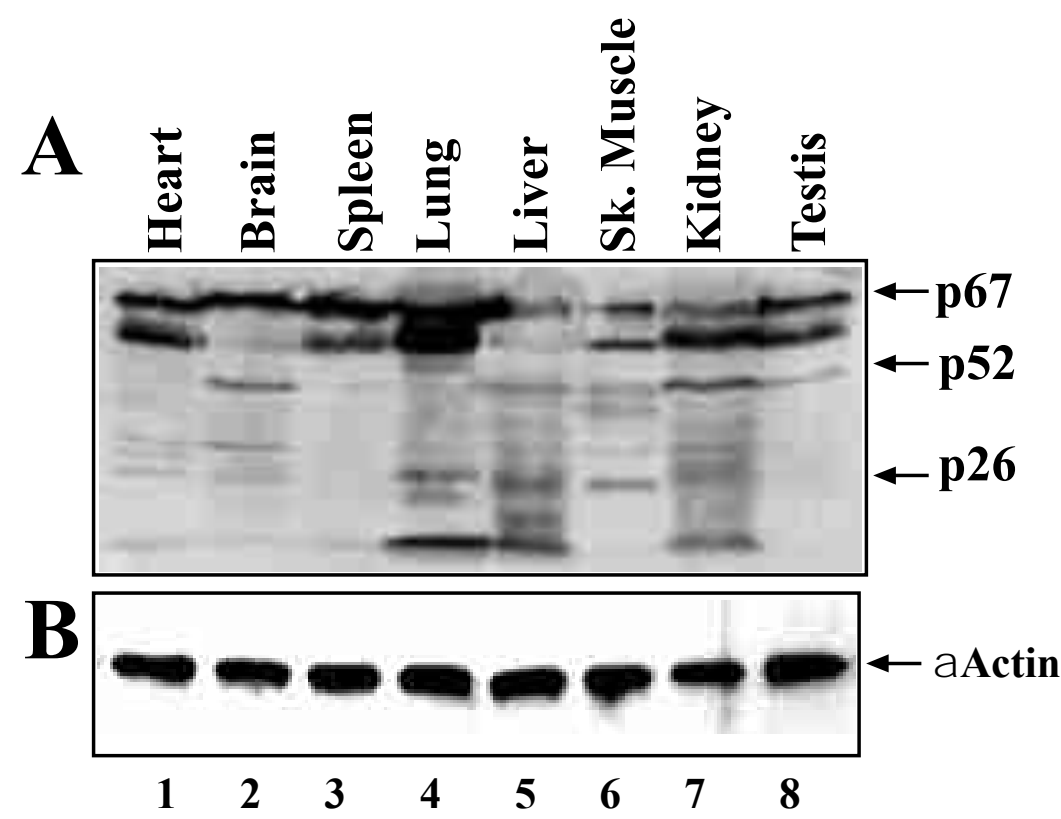

Fig4. Tissue-specific expression of full-length p67 and its segments p26 and p52 male mice

Equal amounts of total protein samples from male mouse tissues as indicated were analyzed on Western blots using polyclonal antibodies specific to p67 (A) and aActin (B) for loading control. This figure is reproduced from Datta and Datta (9) with editor's permission.

\section{IDENTIFICATIONS OF IMPORTANT DOMAINS/MOTIFS/AMINO ACID RESIDUES THAT ARE REQUIRED FOR RAT P67'S AUTO-PROTEOLYSIS IN VITRO}

The His-tagged fusion proteins of p26 and p52 segments of rat p67 were also expressed in Sf21 cells, purified via affinity column chromatography, and their activities were tested in eIF2 $\alpha$ phosphorylation assays (4). We found that p26 segment has significant activity while $\mathrm{p} 52$ has none indicating that p26 segment of p67 is the business end of the molecule and the p52 segment is the regulatory end of protection of eIF $2 \alpha$ phosphorylation activity. In addition, p52 segment alone has auto-proteolysis activity (4). At the p52 segment of p67 there are at least five conserved amino acid residues - D251, D262, H331, E364, and E459 (1-2, 10). This segment upon folding creates a shallow groove where these five amino acid residues juxtapose each other to coordinate with divalent metal ions in vitro (11) and protein domains with positively charged residues such as lysine/arginine residue-rich domains in vitro and in vivo (3). In other word, this shallow groove creates the substrate-binding pocket (11). The strength of the substrate binding may depend upon the H331 residue, which may have destabilization activity of the substrate-enzyme complex. The proper folding of p52 segment also brings H231 site close to the substrate-binding pocket and this residue serves as catalytic site $(3,11)$ that is required for either auto-cleavage or intermolecular cleavage reactions. 
To examine the importance of the above unique and conserved amino acid residues of p67 in intramolecular and/or intermolecular proteolysis reactions, we purified his-tagged p67 point mutants D251A, D262A, H331A, E364A, E459A, and H231E from bacculovirus expression system and tested their auto-proteolysis (4). These purified mutant proteins later passed through several column chromatographic beads including G-50 (molecular sieve), DE52 (anion exchanger), and phosphocellulose (cation exchanger) and eluted bead-bound proteins (wild type or the mutants) by elution buffer containing various salt concentrations such as $0.1 \mathrm{M} \mathrm{KCl}, 0.5 \mathrm{M} \mathrm{KCl}, 0.75 \mathrm{M} \mathrm{KCl}$, and $1.0 \mathrm{M}$ $\mathrm{KCl}$. Eluted proteins were then analyzed on SDS-PAGE followed by Western blotting with an antihistidine antibody. We found that freshly prepared his-tagged full-length rat p67 degraded very slowly in $0.1 \mathrm{M}$ and $0.5 \mathrm{M}$, even in the presence of $1 \mathrm{M}$ urea (4). However, its degradation increased significantly when samples were stored at $-80^{\circ} \mathrm{C}$ over a period of three weeks or more. In addition, p67's degradation was extremely high in elution buffer containing $0.75 \mathrm{M} \mathrm{KCl}$ and after three weeks at $-80^{\circ} \mathrm{C}$, its degradation was down to amino acid level. When $1.0 \mathrm{M} \mathrm{KCl}$ was added to the elution buffer, p67's degradation was at the undetectable level and it did not increase after storage at $-80^{\circ} \mathrm{C}$ over a period of three weeks or more. We then checked the degradation of his-tagged D251A, D262A, H331A, E364A, E459A, and H231E mutants in similar conditions used for full-length p67. We observed quite a good amount of degradation of D251A mutant in all salt concentration, whereas the degradation of D262A and E364A mutants was almost stopped (4). The degradation pattern of E459A mutant was very similar to wild type p67. In contrast, the degradation of H331A mutant was so high we could not recover the full-length mutant. In addition, when this mutant was exogenously expressed in mammalian cells, it not only cleaved itself, it also degraded other cellular proteins including endogenous p67 (4) and other unknown proteins. When we checked the degradation of H231E mutant in elution buffer containing $0.1 \mathrm{M}$ and $0.5 \mathrm{M} \mathrm{KCl}$, we found that it was degraded extensively to generate p26 segment as a major fragmentation product, but at $0.75 \mathrm{M} \mathrm{KCl}$, it generated only the p52 segment. In addition, when this mutant was stored at $-80^{\circ} \mathrm{C}$ for three weeks, we detected mostly the p26 and p52 segments while reducing the full-length p67 in a significant amount. The H231L mutant however showed no degradation at different salt concentration whether it was freshly prepared or stored at $-80^{\circ} \mathrm{C}$ for a longer period of time (4). From these results we concluded that p67 has the autoproteolysis activity and for this activity D262 and E364 residues are absolutely necessary whereas D251 residue for this activity may be redundant. In addition, H331 residue in fact has negative role in p67's auto-proteolysis activity and H231 is the active site for catalysis, where imidazole ring of this histidine is used for electron transfer to cleave the peptide bond. Therefore, substitution of H231 with glutamic acid intensified its electron transfer capability during the cleavage of peptide bonds and high salt like $0.75 \mathrm{M} \mathrm{KCl}$ inhibited this activity.

\section{INTERMOLECULAR PROTEOLYSIS OF RAT P67 IN EX VIVO (CELL CULTURE) SYSTEM}

The H231 residue indeed binds covalently to cell growth inhibitors such as fumagillin and its derivative TNP470 at their epoxy groups (11). This irreversible binding leads to the inhibition of p67's auto-proteolysis activity and this protein accumulates inside the cells. The excess p67 then binds to extracellular signal-regulated kinases $1 \& 2$ (ERK1/2) and inhibits their activation and activity (12) that ultimately leads to the cell-growth inhibition (13-14). Detailed mutagenesis followed by biochemical analyses revealed that a long stretch of amino acid segment starting from 211 to 430 amino acid residues binds to ERK1/2 (15). This segment is not only covering the catalytic H231 site, it is also covering most of the substrate-binding pocket including D251, D262, H331, and E364 residues as well. Therefore, ERK1/2 binding to p67's shallow groove inhibits p67's auto-proteolysis. Indeed, our ex vivo transfection assays show quite a good amount of degradation of Myc-tagged fulllength rat p67 when expressed alone and this degradation was significantly inhibited when $\mathrm{C} 2 \mathrm{C} 12$ myoblasts co-expressed Myc-p67 and Flag-ERK1 or Flag-ERK2 (Fig. 5). P67 also binds to $\alpha$ - and $\gamma$ subunits of eIF2 but not to its $\beta$-subunit (16). Detailed mutagenesis accompanied with extensive biochemical analyses revealed that eIF2 $\alpha$ binds at p67's N-terminal lysine residue-rich domain I $\left.{ }_{36} \mathrm{KKKRRKKKK}_{44}\right)$ and ${ }_{60} \mathrm{SGTS}_{63}$ motif while anchoring at $\gamma$-subunit of eIF2 through its lysine residue-rich domain II $\left({ }_{98} \mathrm{KKKKKKKKKR}_{107}\right)$ and the amino acid segment starting from 310 to 430 amino acid residues $(3,8,16)$. The later segment is also covering a major portion of the substratebinding pocket of $\mathrm{p} 67$. Therefore, $\mathrm{p} 67$ when bound to eIF2, it does not show any intermolecular or intra-molecular degradation. 


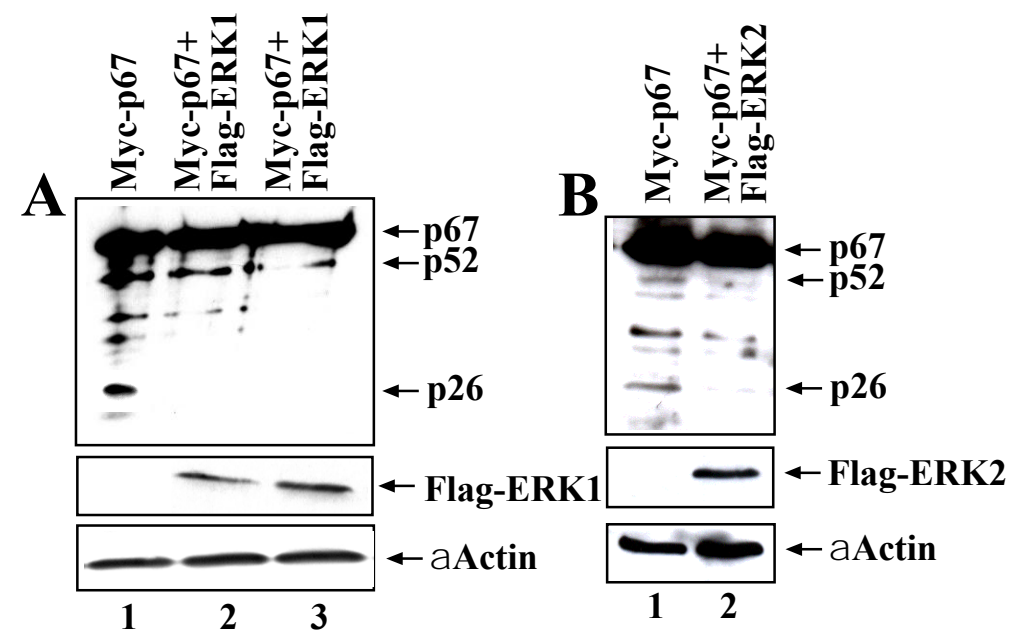

Fig5. Co-expression of Myc-p67 and either flag-tagged ERK1 or ERK2 inhibits the degradation of p67

C2C12 myoblasts were transiently transfected with $5 \mu \mathrm{g}$ of Myc-p67 plasmid alone (lanes 1 in panels A \& B) or in combination with Flag-tagged ERK1 (A) and ERK2 (B). In panel A, lanes 2 \& 3 represent $1 \mu \mathrm{g}$ and $2 \mu \mathrm{g}$ of Flag-tagged ERK1 plasmid and in panel B, lane 2 represents $2 \mu \mathrm{g}$ of Flag-tagged ERK2 plasmid used for transfection assays. After transfection, cell lysates were analyzed on Western blots using monoclonal antibodies specific to His-tag (upper panels in A\& B), Flag-tag (middle panels in A \& B), and aActin (lower panels in A \& $B)$.

\section{Intermolecular Proteolysis Activity Of Rat P67 - Evidence in Ex Vivo SYSTEMS}

If samples of highly purified eIF2 containing its $\alpha-, \beta-, \gamma$-subunits, and p67 were kept frozen in liquid nitrogen for a longer period of time, the $\beta$-subunit of eIF2 disappears (unpublished). This subunit of eIF2 has two lysine residue-rich domains, which are required for the binding to initiator aminoacyltRNA (17). P67's intermolecular auto-proteolysis is mediated by the binding of its lysine residue-rich domains at its substrate-binding pocket (3). During storage conditions, it is possible that a portion of free p67 undergoes auto-proteolysis and generates its p52 segment. Since p52 segment alone has autoproteolysis activity (4), it is more likely that the lysine residue-rich domains of eIF2 $\beta$ binds to the p52 segment and gets cleaved due to p52's intra-molecular proteolysis activity. It may also bind to the full-length p67 at its p52 segment and get cleaved. In support of this view, recently we noticed that another protein, myristoyl alanine-rich C kinase substrate (MARCKS), which has a stretch of lysine residue-rich sequence at its $\mathrm{C}$-terminus, is degraded when p67 was overexpressed in $\mathrm{C} 2 \mathrm{C} 12$ myoblasts (18). In addition, for this cleavage, p67's lysine residue-rich domains and at least D251 and H331 residues are required (18). The N-terminal acidic residue-rich sequences ${ }_{77}$ EEKEKDDDDEDGDGD $_{91}$ ) negatively regulate the functions of lysine residue-rich domains due to the salt-bridge formation (19-20). Once these acidic amino acid residues were substituted with ${ }_{77} \mathrm{QNIQKALEPEAGDGA}_{91}$ residues, the lysine residues are free to coordinate with the acidic residues such as D251, D262, E364, and E459 present in the shallow groove of p52 and stabilize their interactions. This interaction between p26 and p52 segments allows the H231 catalytic site cleaves at the arginine-107 residue and generates p52 segment. Now MARCKS can bind to the shallow groove of p52 segment and gets degraded. Consistent with this, substitutions of D251 and H331 residues with alanine resulted in loss of cleavage of MARCKS. Similarly, the degradation of focal adhesion kinase (FAK) increases as the level of p67 increases during differentiation of $\mathrm{C} 2 \mathrm{C} 12$ myoblasts into myotubes (21) and p67's N-terminal acidic residue-rich sequences and the lysine residue-rich sequences play important roles in this degradation process. Substitutions of either of acidic residuerich sequences or lysine residue-rich sequences of p67 lead to the complete degradation of FAK (21). We also observed similar results for the degradation of Cdc42 (22), which is a Rho GTPase and important roles of its N-terminal domains of p67. Cdc42 in fact is detected in $\mathrm{C} 2 \mathrm{C} 12$ myoblasts as two forms - one is the slower migrating "pro-form" and the other is the matured form. Overexpression of wild type rat p67 in rat tumor hepatoma cells increased both forms of Cdc42 slightly as compared to control cells expressing EGFP alone, whereas expression of either D6/2 ${ }_{77}$ EEKEKDDDDEDGDGD $_{91}$ changed to ${ }_{77} \mathrm{QNIQKALEPEAGDGA}_{91}$ ) or K1K2 mutant degraded the 
"pro-form" completely and resulted into more accumulation of the mature Cdc42 (22). In contrast, expression of D251A and D262A mutants showed no significant degradation of the pro-Cdc42 (22). These results suggest that either acidic residue-rich domain or lysine residue-rich domains I \& II or all of these domains are stabilizing the "pro-form" of Cdc42. P67 is also involved in overexpression of protein kinase $\mathrm{C}(\mathrm{PKC})$ in $\mathrm{C} 2 \mathrm{C} 12$ myoblasts (23) and p21-activating kinase 1 (Pak1) in $\mathrm{C} 2 \mathrm{C} 12$ myotubes (22). At present it is not clear however whether these effects on degradation/maturation of MARCKS, FAK, and Cdc42 due to constitutive expression of rat p67 or its certain mutants in $\mathrm{C} 2 \mathrm{C} 12$ myoblasts are direct or indirect. Constitutive expression of rat p67 in KRC-7 cells reduced the level of PERK, an eIF2 $\alpha$-specific kinase, and its level increased when these cells were overexpressing D251A mutant (24). It is however not clear at present how p67 can show differential effects on degradation or stabilization of different cellular proteins. Nonetheless, one thing is clear from the above evidence that p67 is involved in intra-molecular proteolytic activities of certain proteins while stabilizing the other proteins possibly by degrading some inhibitory proteins.

To examine whether p67's proteolytic activity is involved in the degradation of cell cycle regulatory proteins such as cyclin and Cdks, we constitutively expressed wild type rat p67 and its mutants D251A, H231E, D6/2, and K1K2 in C2C12 myoblasts (25). Degradation of cyclins and Cdks are essential for the transition of one phase of the cell cycle to the next (26-27). Levels of the different cyclins such as cyclin D1, cyclin E, cyclin A, and cyclin B1 and Cdks such as Cdk1, Cdk2, Cdk4, and Cdk6 were examined in Western blots. We found that p67 is involved in lowering the overall concentrations of cyclin D1/Cdk4 and cyclin D1/Cdk6 complexes. It is involved in the maturations of "pro forms" of cyclins E, A, and B1 but not cyclin D1. Its D251 and H231 amino acid residues and the $\mathrm{N}$-terminal acidic residue-rich sequences are involved in this maturation process. Degradation of cyclin B1 in p67-expressing cells was very extensive and we found that p67 indeed binds to cyclin B1 in our co-immuno-precipitation assays (25). Generation of the "pro-forms" of proteins due to the expression of D251A mutant is not limited to some of the cyclins, this mutant along with H331A and E364A mutants also generate "pro-form" of p21 (Fig. 6), the cell cycle inhibitory protein, whose expression in cells could be p53-dependent or independent. Overexpression of wild type rat p67 and its mutants D262A and E459A in rat tumor hepatoma cells processed the "pro-form" of p21 completely or near completely (Fig. 6). These results suggest that proteolytic processing of "proform" of p21 to its mature form needs D251, H331, and in part E364 amino acid residues of p67. Proform of a protein is the longer version of the mature form. How does it originate during translation is an open question. Irrespective of the mechanisms of generation of "pro-forms" of certain cyclins and $\mathrm{p} 21$, it is clear that $\mathrm{p} 67$ 's proteolytic activity is indeed involved in the maturation of certain cyclins, $\mathrm{p} 21$, and possibly several other unidentified cellular proteins.

\section{Rat Tumor Hepatoma Cells expressing}

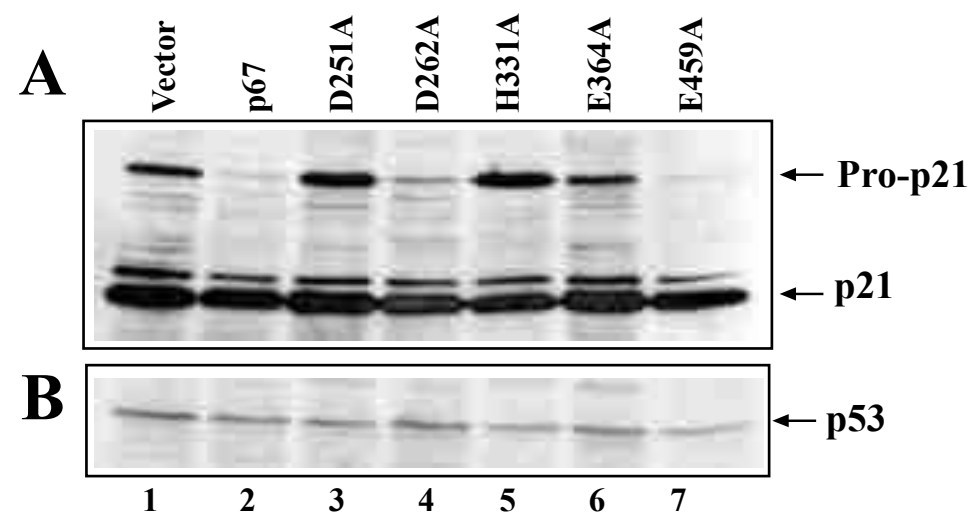

Fig6. Maturation of p21 in rat tumor hepatoma (KRC-7) cells constitutively expressing EGFP alone (Vector) or its fusions with wild type rat 667 and its point mutants (D251A, D262A, H331A, E364A, and E459A)

Equal amounts of cell lysates from different lines were prepared and analyzed on Western blots using rabbit polyclonal antibodies (Sc-471; M-19 from Santa Cruz Biotechnology) specific to cell cycle inhibitor p21 (A) and a monoclonal antibody specific to p53 (Ab-5, Calbiochem) (B), the tumor suppressor.

\section{CONCLUDING REMARKS AND FUTURE DIRECTIONS}

P67 shares more than 95\% sequence identity within mouse, rat, and human. The p52 segment shares near $100 \%$ sequence identity within mammals, whereas the amino acid sequences of the p26 segment 


\section{Bansidhar Datta}

are somehow diverse (1-3). Comparison of the promoter sequences within mouse, rat, and human showed quite a significant overlap within cis-elements and trans-acting factors that regulate the expression and activity of p67 genes from these species (28). Detailed mutagenesis accompanied with extensive biochemical analysis in in vitro, ex vivo, and in vivo conditions should be carried out to define the importance of the various cis-elements during tissue-specific and species-specific expression of the $\mathrm{p} 67$ genes.

Detailed mutagenesis followed by biochemical analyses of rat p67 revealed functional domains/motifs that are important for the regulation of phosphorylation of eIF2 $\alpha$, ERK1/2 MAP kinases, possibly other kinases, and intra-molecular and intermolecular proteolytic activities. The targets for p67's intermolecular proteolytic activity are cell cycle regulatory proteins, certain cyclins and Cdks, and p21; cell growth, cytoskeleton dynamics, migration, and motility regulatory proteins, PKC, MARCKS, FAK, Cdc42, PAK1, and Akt1/2 (or PKB); eIF2 $\alpha$-specific kinase, PERK; and possibly several other unidentified kinases and phospho-proteins that are involved in cell signaling. In addition to PERK, it remains to be examined whether other eIF2 $\alpha$-specific kinases such as HRI, PKR, and mammalian homolog of yeast GCN2 are the targets for p67's inter-molecular proteolytic activity. It also remains to be examined how many of these targets are involved in direct or indirect proteolysis reactions. During the course of our studies, we have identified several dominant negative and dominant positive p67 mutants - some of them are single point mutants, some of the them are block mutants, and a few of them are double mutants i. e. combination of a block mutant and a single point mutant. These mutants are serving important roles in defining the role(s) of p67 in various cellular processes. It would be interesting to identify some of these mutants in disease conditions as well.

\section{REFERENCES}

[1] Datta, B. (2000) MAPs and POEP of the Roads from Prokaryotic to Eukaryotic Kingdoms - A Review. Biochimie 82, 95-107.

[2] Datta, B. (2009) Roles of P67/MetAP2 as a tumor suppressor - a review. Biochim. Biophys. Acta 1796, 281-292.

[3] Datta, B. (2015) Diversified roles of p67/MetAP2 as a regulator of cell growth and differentiation, in tumor suppression, and in obesity. Review, Curr. Topics Biochem. Res. 16, 41-52.

[4] Datta, B., Ghosh, A., Majumdar, A., and Datta, R. (2007) Auto-proteolysis of rat p67 generates several peptide fragments: the N-terminal fragment, $\mathrm{p} 26$, is required for the protection of eIF2 $\alpha$ from phosphorylation. Biochemistry 46, 3465-3475.

[5] Clinkinbeard, T., Ghoshal, S., Craddock, S., Pettigrew, L. C., Guttmann, R. P. (2013) Calpain cleaves methionine aminopeptidase-2 in a rat model of ischemia/reperfusion, Brain Res. 1499, 129-135.

[6] Ghosh, A., Tammali, R., Balusu, R., Datta, R., Chattopadhyay, A., Bhattacharya, M., and Datta, B. (2014) Oligomerization of the eukaryotic initiation factor 2-associated glycoprotein p67 requires N-terminal 1-107 amino acid residues. Intl. J. Appl. Biotech. Biochem._4, 25-44.

[7] Datta, B., Ray, M.K. Chakrabarti, D. Wylie, D. E and Gupta, N.K. (1989) Glycosylation of Eukaryotic Peptide Chain Initiation Factor 2 (eIF-2)-associated $67 \mathrm{kDa}$ Polypeptide $\left(\mathrm{p}^{67}\right)$ and Its Possible Role in the Inhibition of eIF-2 Kinase-catalyzed Phosphorylation of the eIF-2 $\alpha$-Subunit by eIF-2. J. Biol. Chem. 264, 20620-20624.

[8] Datta, R., Choudhury, P., Ghosh, A., and Datta, B. (2003) A glycosylation site, ${ }_{60} \mathrm{SGTS}_{63}$, of p67 is required for its ability to regulate the phosphorylation and activity of eukaryotic initiation factor2 alpha (eIF2 $\alpha)$. Biochemistry 42, 5453-5460.

[9] Datta, B. and Datta, R. (2016) Analysis of p67 expression in different mouse tissues. Intl. J. Adv. Res. Chem. Sci. 3, 20-25.

[10] Wu, S., Gupta, S., Chatterjee, N., Hileman, R.E., Kinzy, T.G., Denslow, N.D., Merrick, W.C., Chakrabarti, D., Osterman, J.C., and Gupta, N.K. (1993) Cloning and characterization of complementary DNA encoding the eukaryotic initiation factor 2-associated 67-kDa protein $\left(\mathrm{p}^{67}\right)$ J. Biol. Chem. 268, 10796-10801.

[11] Liu, S., Widom, J., Kemp, C. W., Crews, C. M., and Clardy, J. (1998) Structure of human methionine aminopeptidase-2 complexed with fumagillin. Science 282, 1324-1327. 
[12] Datta, B., Majumdar, A., Datta, R., and Balusu, R. (2004) Treatment of cells with the angiogenic inhibitor fumagillin results in increased stability of eukaryotic initiation factor 2 -associated glycoprotein, p67 and that inhibits the phosphorylation of extracellular signal-regulated kinases. Biochemistry 43, 14821-14831.

[13] Meloche, S., and Pouyssegur, J. (2007) The ERK1/2 mitogen-activated protein kinase pathway as a master regulator of the G1- to S-phase transition. Oncogene 26, 3227-3239.

[14] Katz, M., Ido, A., and Yarden, Y. (2007) Regulation of MAPKs by growth factors and receptor tyrosine kinases. Biocheim. Biophys. Acta 1773, 1161-1176.

[15] Majumdar, A., Ghosh, A., Datta, S., Prudner, B., and Datta, B. (2010) P67/MetAP2 suppresses K-RasV12 mediated transformation of NIH3T3 mouse fibroblasts in culture and in athymic mice. Biochemistry 49, 10146-10157.

[16] Ghosh, A., Datta, R., Majumdar, A., Bhattacharya, M., and Datta, B. (2006) The N-terminal lysine residue-rich domain II and the 340-430 amino acid segment of eukaryotic initiation factor 2-associated glycoprotein p67 are the binding sites for the $\gamma$-subunit of eIF2. Exp. Cell Res. 312, 3184-3203.

[17] Laurino, J. P., Thompson, G. M., Pacheco, E., and Castilho, B. A. (1999) The $\beta$ subunit of eukaryotic initiation factor 2 binds mRNA through the lysine repeats and a region comprising the C2-C2 motif. Mol. Cell. Biol. 19, 173-181.

[18] Datta, B, and Datta, R. (2016) Differential expression of MARCKS in C2C12 myoblasts and myotubes constitutively expressing p67. Int. J. Adv. Res. Chem. Sci. 3(2), 15-22.

[19] Datta, R., Tammali, R., and Datta, B. (2003) Negative regulation of the protection of eIF2 $\alpha$ phosphorylation activity by a unique acidic domain present at the N-terminus of p67. Exp. Cell Res. 283, 237-246.

[20] Datta, B., Datta, R., Ghosh, A., and Majumdar, A. (2006) The binding between p67 and eukaryotic initiation factor 2 plays important roles in the protection of eIF2 $\alpha$ from phosphorylation by kinases. Arch. Biochem. Biophys. 452, 138-148.

[21] Datta, B. and Datta, R. (2016) The N-terminal acidic residue-rich domain and lysine-rich domains I and II of p67 are required for stable expression of focal adhesion kinase (FAK). Int. J. Adv. Res. Chem. Sci. 3(3):1-6

[22] Datta, B. and Datta, R. (2016) P67 is involved in the regulation of cytoskeleton dynamics by modulating the expression and activity of Pak1 in differentiated $\mathrm{C} 2 \mathrm{C} 12$ myoblasts. Int. J. Adv. Res. Chem. Sci. 3(4), 1-8.

[23] Datta, S. K. and Datta, B. (2016) Constitutive expression of p67 increases cPKCalpha level in C2C12 myoblasts and decreases in myotubes. Int. J. Adv. Res. Chem. Sci. 3(2), 9-14.

[24] Datta, B., Datta, R., Ghosh, A., and Majumdar, A. (2004) Eukaryotic initiation factor 2associated glycoprotein, p67, shows differential effects on the activity of certain kinases during serum-starved conditions. Arch. Biochem. Biophys. 427, 68-78.

[25] Datta, S. K. and Datta, B. (2016) P67 shows differential effects in proteolysis and maturation of cell cycle regulatory proteins, cyclins and Cdks. Intl. J. Adv. Res. Chem. Sci. 3, 1-10.

[26] Malumbres, M. (2014) Cyclin-dependent kinases. Genome Biol. 15, 122-131.

[27] Lim, S., and Kaldis, P. (2013) Cdks, cyclins and CKIs: roles beyond cell cycle regulation. Development 140, 3079-3093.

[28] Datta, B., Earl, D., Roods, M., Datta, S. (2014) Analysis of p67/MetAP2 gene from mouse, rat, and human. Intl. J. Mol. Genet.5, 1-12.

\section{AUTHOR's BIOGRAPHY}

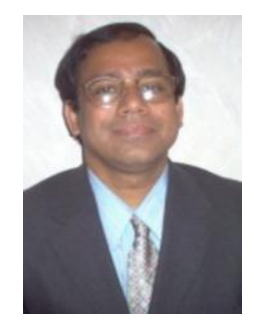

Bansidhar Datta, Ph.D., Associate Professor, Department of Chemistry \& Biochemistry, Kent State University, Kent, OH 44240, USA 